\title{
Shaping Current Waveforms for direct Modulation of Semiconductor Lasers
}

\author{
Lucas Illing and Matthew B. Kennel
}

\begin{abstract}
We demonstrate a technique for shaping current inputs for the direct modulation of a semiconductor laser for digital communication. The introduction of shaped current inputs allows for the suppression of relaxation oscillations and the avoidance of dynamical memory in the physical laser device, i.e., the output will not be influenced by previously communicated information. On the example of time-optimized bits, the possible performance enhancement for high data rate communications is shown numerically.
\end{abstract}

Index Terms - Semiconductor lasers, Intersymbol interference, Time optimal control, Optimal control, Communication system nonlinearities, Nonlinear optics, Nonlinear systems

\section{INTRODUCTION}

$\mathbf{S}$ EMICONDUCTOR lasers typically convey information for optical communication using either "external" or "direct" modulation. In the first scheme, the laser is maintained in a constant light-emitting state and some sort of external "shutter," typically a Mach-Zehnder type electro-optic modulator, modulates the output intensity according to an externally applied voltage. The advantage of this technique is that it allows high bit rates $(>10 \mathrm{Gbit} / \mathrm{s})$ and there is no interaction between the information being applied to the modulator and any internal dynamics of the laser. The disadvantage is that costly external apparatus are necessary. Direct modulation involves changing the current input of the laser, i.e., modulating it around the bias current, in order to produce time-dependent output in optical intensity.

In terms of modeling, the use of direct modulation means that the laser becomes a driven nonlinear system with potentially complicated dynamics. Indeed, it is well known that even simple high-frequency periodic modulation of sufficiently large amplitude can lead to nonlinear phenomena such as period doubling cascades, period tripling, and chaos [1]-[8]. It is therefore not surprising that attempting communication with current pulses that occur rapidly in succession, quickly changing among discrete current levels to transmit randomly interleaved zero and one bits, will result in a complex output time series of intensity. As a result the bits may cease to be decodable at the receiver and there is a considerable memory effect [9]. This effect is called "inter-symbol interference" in the communications literature because the sequence of

This work was supported in part by the US Army Research Office (grant \# DAAD19-99-1-0199 and grant \# DAAG55-98-1-0269)

L. Illing is with the Department of Physics and the Center for Nonlinear and Complex Systems, Duke University, P.O. Box 90305, Durham, NC 27708 (e-mail:illing@phy.duke.edu)

M. B. Kennel is with the Institute for Nonlinear Science, University of California, San Diego, La Jolla, CA 93093-0402 information symbols at previous times may harm decoding at the present time. Additionally, communication using highspeed direct modulation is limited by transient oscillations, known as relaxation oscillations, that result from the interplay between the optical field and the carrier density [10], [11]. Such oscillations cause distortions of the output light pulse and broaden the signal's optical spectrum (increased chirp), thus contributing to an increased bit-error rate.

In an effort to overcome the problems associated with direct modulation many techniques for chirp reduction and suppression of relaxation oscillations have been proposed, including the modification of the physical laser device [11] [13], the use of external optical feedback [14] and external electronic circuits [15], as well as the shaping of the current pulses that are used to encode logical bits into the optical output of the laser [16]-[20].

We approach the issue of inter-symbol interference and relaxation oscillations from the point of view of nonlinear dynamics. A basic single-mode semiconductor laser modeled by the rate equations is a two degree of freedom system, with ordinary differential equations for photon density and carrier density. The cause of inter-symbol interference is the fact that even if the observable intensity returns to its nominal stationary condition after a current wave form is applied, the unobservable carrier density may not have, and thus there is dynamical coupling from past to future. Our feed-forward solution is to use appropriately shaped driving currents to control the state-space trajectory of the laser, and we show how to obtain these waveforms given a dynamical model for the laser.

The main goal of this paper is to demonstrate a technique that can eliminate relaxation oscillations and intersymbol interference, without having to physically change the laser diode. This allows enhanced data rates and improved communication performance as is shown numerically for a communication scheme using time-optimized non-return-tozero (NRZ) bits. This improvement comes at the cost of more complex input electronics, which would be necessary to shape the input current in experiments. A distinguishing feature of our approach is the use of shaped, continuous, drive currents with finite rise times. This contrasts with other recent research [20], where relaxation oscillations are suppressed using piece-wise constant currents, which would possibly present difficult discontinuities.

We will first present the general idea of the shaping method in Sec. II and then show particular solutions for the specific case of optimizing the transition time for NRZ bits in Sec. IIII Using these solutions, we estimate the bit error rate (BER) 
performance for the case of a simple Gaussian white noise channel to show the possible performance enhancement.

\section{Current Shaping}

The semiconductor laser is presumed to be single mode. For this case the laser dynamics can be described by a driven two dimensional system of ordinary differential equations for the carrier density $N(t)$ and the photon density $S(t)$, the latter being proportional to output intensity:

$$
\begin{aligned}
\frac{d S}{d t} & =-\gamma_{c} S+\Gamma G(N, S) S, \\
\frac{d N}{d t} & =-\gamma_{s} N-G(N, S) S+\frac{J_{0}+J}{e d} .
\end{aligned}
$$

The constant $J_{0}$ is the bias current, $J(t)$ the modulation around the bias, $G(N(t), S(t))$ the optical gain coefficient including nonlinear effects, $\Gamma$ the confinement factor, $d$ the active layer thickness of the laser, $\gamma_{c}$ the photon decay rate, and $\gamma_{s}$ the spontaneous carrier decay rate.

For the purpose of numerical calculations and analytical investigations it is useful to work with dimensionless quantities. It is convenient to non-dimensionalize using the fixed point $\left(S_{0}, N_{0}\right)$ of the laser in the absence of modulation $(J(t)=0)$, that is, of the laser under stationary continuous-wave operating conditions $(d S / d t=d N / d t=0)$. We expand the nonlinear $G(N(t), S(t))$ around this point to first order

$$
G(N, S)=G_{0}+G_{n}\left(N-N_{0}\right)+G_{p}\left(S-S_{0}\right) .
$$

Liu and Simpson [21] show how to experimentally estimate $G_{n}=\partial G / \partial N>0$ and $G_{p}=\partial G / \partial S<0$. Note that the fixed point conditions yield the relations $\Gamma G_{0}=\gamma_{c}$ and $J_{0} / e d-$ $\gamma_{s} N_{0}=G_{0} S_{0}$.

These dimensionless dynamical equations are independent of how far above threshold the laser is operated except via indirect influence on the empirical differential gain parameters $G_{n}$ and $G_{p}$, which depend on the expansion point. The dimensionless photon density $s(t)$ is defined via $s(t)=S(t) / S_{0}$, the carrier density $n(t)$ via $n(t)=N(t) / N_{0}-1$, and the current via $\mathcal{J}+\mathcal{J}_{\mathcal{M}}=\left(J_{0}+J(t)\right) /\left(\gamma_{s} N_{0} e d\right)-1 .{ }^{1}$ The dimensionless gain $g=G / G_{0}$ is

$$
g(n, s)=1+\frac{\gamma_{n}}{\mathcal{J} \gamma_{s}} n-\frac{\gamma_{p}}{\gamma_{c}}(s-1),
$$

where $\gamma_{n}=G_{n} S_{0}$ and $\gamma_{p}=-\Gamma G_{p} S_{0}$. Using the natural relaxation oscillation angular-frequency $\omega_{R}=\sqrt{\gamma_{c} \gamma_{n}+\gamma_{s} \gamma_{p}}$, the dimensionless quantity corresponding to time $\tau=t \omega_{R}$ can be formed. With this the model equations are:

$$
\begin{aligned}
& \frac{d s}{d \tau}=\frac{\gamma_{c}}{\omega_{R}}([g(n, s)-1] s) \\
& \frac{d n}{d \tau}=\frac{\gamma_{s}}{\omega_{R}}\left(\mathcal{J}+\mathcal{J}_{\mathcal{M}}-n-\mathcal{J} g(n, s) s\right) .
\end{aligned}
$$

Table \ lists the dimensionless quantities and the parameters we use, the values of which are based on measurements of J.M. Liu's group [22]. For the purpose of digital communication

\footnotetext{
${ }^{1}$ Roughly speaking, the dimensionless total current $1+\mathcal{J}+\mathcal{J}_{\mathcal{M}}$ is in units of the threshold current. This is strictly true for simple gain functions that imply clamping of the carrier density to it's threshold value for currents above threshold.
}

TABLE I

DYNAMICAL VARIABLES, DEFINITIONS, AND NUMERICAL VALUES OF PARAMETERS

\begin{tabular}{c|c|l}
\hline Symbol & Value & Description \\
\hline$s(\tau)$ & & dimensionless photon density \\
$n(\tau)$ & & dimensionless carrier density \\
$g(n, s)$ & & dimensionless gain coefficient \\
$\mathcal{J}_{\mathcal{M}}(\tau)$ & & dimensionless modulation current \\
\hline $\mathcal{J}$ & $2 / 3$ & bias current at fixed point \\
$\gamma_{s}$ & $1.458 \times 10^{9} s^{-1}$ & spontaneous carrier decay rate \\
$\gamma_{n} / \mathcal{J}$ & $2.0 \times 10^{9} s^{-1}$ & gain variation with carrier density \\
$\gamma_{p} / \mathcal{J}$ & $3.6 \times 10^{9} s^{-1}$ & gain variation with photon density \\
$\gamma_{c}$ & $3.6 \times 10^{11} s^{-1}$ & photon decay rate \\
$\omega_{R}$ & $\sqrt{\gamma_{c} \gamma_{n}+\gamma_{s} \gamma_{p}} \sim \frac{1}{45 \mathrm{ps}}$ & relaxation oscillation frequency \\
\hline
\end{tabular}

we are interested in the relationship between the information bearing modulation current and the output of the laser. The key insight is to note that, given a functional form for the time series $s(t)$, the corresponding input current can be computed just by differentiation. In contrast, given the functional form of the current, it is necessary to integrate the nonlinear equations of motion (4) to obtain the potentially chaotic resulting laser output.

Given a particular model for the laser, there is often some differential operator taking a given $s(\tau)$ to the other variables $O_{s}[s(\tau)]=\mathcal{J}_{\mathcal{M}}(\tau)$ and another one $O_{n}[s(\tau)]=n(\tau)$ such that the state vector $\left[\mathcal{J}_{\mathcal{M}}(\tau), n(\tau), s(\tau)\right]$ is a solution to the equations of motion. This solution though may not be physically allowable (such as having negative total current) or dynamically stable.

To derive the driving current as function of the output waveform we introduce, for computational ease, $s(\tau)=e^{y(\tau)}$, solve for $n(\tau)$ using the $d s / d \tau$ equation, differentiate, and rearrange the $d n / d \tau$ equation to solve for $\mathcal{J}_{\mathcal{M}}(\tau)$, yielding

$$
\mathcal{J}_{\mathcal{M}}=\frac{\omega_{R}^{2} \mathcal{J}}{\gamma_{c} \gamma_{n}}\left[\ddot{y}+\left(\frac{\gamma_{p}+\gamma_{n}}{\omega_{R}} e^{y}+\frac{\gamma_{s}}{\omega_{R}}\right) \dot{y}+\left(e^{y}-1\right)\right] \text {. }
$$

This equation relates a given waveform $s(\tau)$, via $y$, to the modulation current that caused it. Any desired output waveform that corresponds to a physically allowed input can thus be produced by driving the laser with the according shaped current $\mathcal{J}_{\mathcal{M}}(\tau)$. Not all functions $s(\tau)$ will however result in realistic $\mathcal{J}_{\mathcal{M}}(\tau)$. One physical requirement is a non-negative total driving current at all times, $1+\mathcal{J}+\mathcal{J}_{\mathcal{M}} \geq 0$, as it is not possible to extract electrical energy back from the carrier density. Practical considerations generally limit the peak current that can be applied as well.

Note that (5) includes the full nonlinear dynamics of the laser as modeled by the rate equations (11. In contrast to any theory based on the linearization of $\mathbf{1}$, results obtained using (5) apply to large driving amplitudes. The specific linearized parameterization of gain we used (2) restricts the validity of (4) because it does not take into account gain saturation effects that become important for very large intracavity photon densities $s$. However, it is equally straightforward to derive 
a)

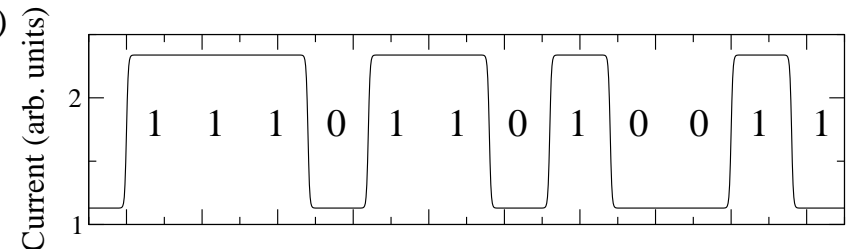

b)

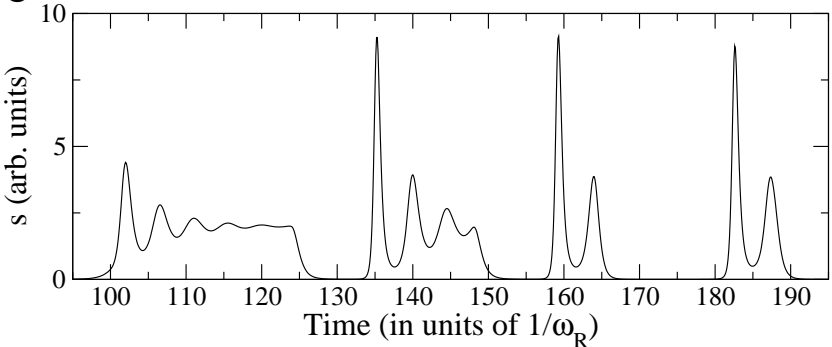

Fig. 1. We show how a square-like total driving current $1+\mathcal{J}+\mathcal{J}_{\mathcal{M}}$ (a) gives rise to relaxation oscillations of the laser photon density $s(t)$ (b). The bit time is $T_{b i t}=8$ which corresponds to $360 \mathrm{ps}$ for the model-parameters shown in Tab. 【 $\left(\omega_{R}^{-1} \sim 45 \mathrm{ps}\right)$. The lower and upper value for the driving current corresponds to fixed points with $s=0.2$ and $s=2.0$, respectively.

formulas analogous to 5 when gain functions which model saturation are used. We chose to work with gain 2 because it is simple. For the results presented in the rest of the paper gain saturation is not important, and, the parameters of (2) may be estimated experimentally.

\section{Application to COMmunication}

We consider a "direct" modulation setup: the laser's driving current $J(t)$ is modulated to encode information. The laser light travels through the communication channel, and at the receiver, the intensity of the arriving signal is measured. One then attempts to extract the information from this signal, in the simplest case by a single thresholding to decode binary information.

Figure 1 shows the laser dynamics driven with an unshaped current waveform (NRZ bits). After a transition from low current to high current, there are clear relaxation oscillations of the laser output, that is, the output "rings" at the characteristic relaxation oscillation frequency. In the particular situation here, the bit time would be sufficiently large that a conventional system could decode the information successfully by integrating energy over a bit interval. A further increase in bit rate, however, will result in significant inter-symbol interference (see Fig. 3 $\mathrm{d}$-f)

The state-space trajectory depends on its initial conditions $s^{0}$ and $n^{0}$ as well as on the driving signal $\mathcal{J}_{\mathcal{M}}(\tau)$. If, at the end of each bit time $T_{b i t}$, the system returns to a particular point in state-space, e.g. $s\left(T_{b i t}\right)=s^{0}$ and $n\left(T_{b i t}\right)=n^{0}$, then repeating the same driving signal $\mathcal{J}_{\mathcal{M}}(\tau)$ will result in a repetition of the state-space trajectory. If, on the other hand, the system trajectory did not reach $\left(s^{0}, n^{0}\right)$ after the first bit, then repeating the driving signal will result in a different laser output, whose particular details depend on the previous drive signal. This dynamic coupling causes intersymbol interference.

The main physical issue is to return the unobserved carrier density to a specific state as well as the photon density. We use (5) to design driving signals which return the system trajectory to a known state at the end of each bit. Since the adverse effects of dynamic coupling on communication performance are especially relevant for high bit rates, we will try to maximize the bit rate (minimizing the bit time) for a given physical laser by optimizing driving signals which cause no dynamical inter-symbol interference.

\section{A. Finding the time-optimal NRZ bits}

NRZ bits encode 1-bits (0-bits) as high (low) light power, but in contrast to return-to-zero pulsing, the intensity stays high when two or more consecutive 1-bits are transmitted, and similarly for consecutive zero bits. The dynamical picture is a system that can be driven to switch between two fixed points in state-space, one corresponding to low output power, the "off" state, and the other corresponding to high output power, the "on" state. The driving waveform for a bit identical to its predecessor is trivial - a constant to stay at the particular fixed point. We solve for driving currents that fix the system along a state-space trajectory, smoothly connecting the "off" fixed point to the "on" fixed point (up transition) and another one for the "on" to "off" transition (down transition). We require both the photon density and the carrier density come to a known fixed point at the end of each bit, thus there will be no dynamic coupling from one bit to the next and therefore no inter-symbol interference.

We use 5, a parameterized set of photon density wave forms, and an optimization algorithm to search for waveforms which optimize some other desirable communication criterion under the condition that they correctly connect the two states. In particular, we have searched for the minimum transition time $T$, and thus highest bit rate, given a desired intensity separation between high and low.

To be specific, let us fix the "off" intensity to $20 \%$ of the fixed point intensity around which we normalized, $s_{\text {off }}=0.2$, and the "on" state to twice the fixed point value, $s_{\text {on }}=2.0$. For the up (down) transition we need to search the space of all smooth waveforms $s(\tau)$ with $s(0)=s_{\text {off }}$ and $s(T)=s_{\text {on }}$ $\left(s(0)=s_{\text {on }}\right.$ and $\left.s(T)=s_{\text {off }}\right)$ such that the transition time $T$ is minimized and such that the corresponding current $\mathcal{J}_{\mathcal{M}}(\tau)$ meets certain constraints. In this example, we require that $0<$ $1+\mathcal{J}+\mathcal{J}_{\mathcal{M}}<6$, which means that the total input current into the laser is positive and that the largest pump current we admit is roughly six times the threshold current, a physically reasonable requirement. We furthermore require that $\mathcal{J}_{\mathcal{M}}(\tau)$ has zero first derivatives at $\tau=0$ and $\tau=T$.

The success of the optimization over all possible waveforms depends on the choice of the right set of basis functions, and satisfying all the boundary conditions. We found that, although an ordinary Fourier base does work to a certain extent, it is numerically advantageous to pick base functions which automatically incorporate many of the constraints, avoiding needing as many explicit constraint equations in the optimization procedure. This notion led us to use the following functions to parameterize $s=e^{y}$ :

$$
y(\tau)=p\left(\frac{\tau}{T}\right)+\left[\sum_{k=1}^{N} a_{k} \sin \left(2 \pi f\left(\frac{\tau}{T}\right)\right)\right]^{4},
$$




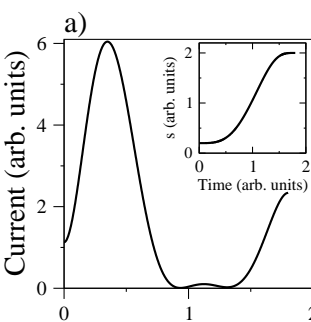

b)

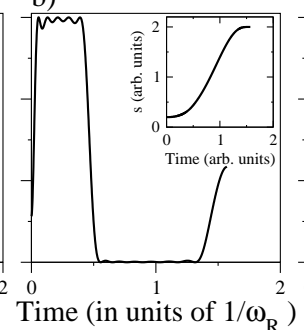

c)

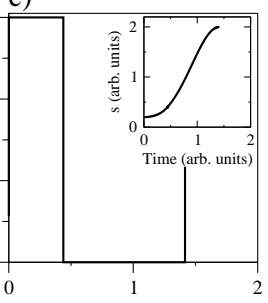

e)

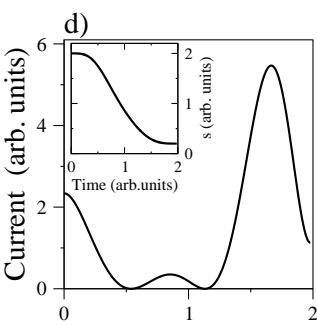

e)

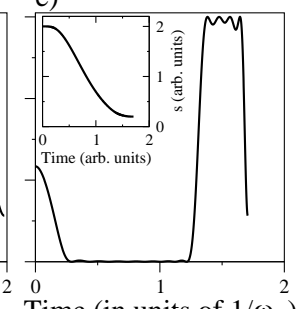

Time (in units of $1 / \omega_{\mathrm{R}}$ )

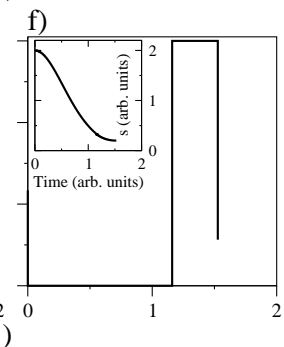

Fig. 2. The figure shows driving current waveforms, i.e. $1+\mathcal{J}+\mathcal{J}_{\mathcal{M}}(t)$, and in the insets the corresponding optical laser output in terms of the photon density $s(t)$. Panels a), b), and c) show the transition from the "off" to the "on" state. Using two sin-terms $[\mathrm{N}=2$ in [6] in the optimization procedure for the driving current yields a), whereas the use of 20 base functions $(\mathrm{N}=20)$ results in b). For comparison, we display in c) the photon density waveform and discontinuous current computed using optimal control theory. Panels d), e), and f) are results for the "on" to "off" transition, where two base functions are used in d), 20 base functions in e), and f) is the optimal control theory result.

where $p(\cdot)$ is a (7th degree) polynomial with $p(0)=\ln [s(0)]$, $p(1)=\ln [s(T)]$, and with zero first, second, and third derivative at $\tau=0$ and $\tau=T$, which ensures zero first derivatives of $\mathcal{J}_{\mathcal{M}}$ at these times. $f(x)=\left(a_{0}+1\right) x /\left(a_{0} x+1\right)$ is a warp function introducing asymmetry with the effect that short transition times can be obtained using fewer base functions. The only constraint that has to be enforced in the optimization is the requirement $0<1+\mathcal{J}+\mathcal{J}_{\mathcal{M}}<6$ (see Table $\Pi$. This choice of base functions was rather arbitrary, justified principally by numerical ease in automatically incorporating boundary conditions and empirically giving reasonably successful results, not by any strong theoretical considerations. We leave the question of how to choose mathematically optimal, or experimentally desirable, base functions for future investigations. Our main goal is to demonstrate the general concept; there is no barrier to incorporating other forms of base functions and other models for different lasers in this method.

Figure 2 shows numerical results from using base functions (6) and the NPSOL software package [23], [24], which allows nonlinear constrained optimization. Comparing the currents for the "off" to "on" transition (Fig. 2a, b, and c) to the corresponding photon densities $s(t)$ (insets) shows that the shaped current waveform that is obtained as result of the optimization is quite dissimilar from the output intensity waveform.

In particular, an increase of the laser's output intensity is not achieved by just ramping up the current from the low value corresponding to the "off" steady state to the higher value corresponding to the "on" state. Instead the pump current is reduced after an initial surge to allow the carrier density to
TABLE II

SUMMARY OF PARAMETERS, CONSTRAINTS, AND RESULTS FOR THE OPTIMIZED NRZ BITS

\begin{tabular}{|c|c|c|c|}
\hline \multicolumn{4}{|c|}{ Fixed parameters in the optimization } \\
\hline \multicolumn{2}{|l|}{ ed point } & \multicolumn{2}{|c|}{$s_{0 b i t}=0.2$} \\
\hline \multicolumn{2}{|l|}{ 1-bit fixed point } & \multicolumn{2}{|c|}{$s_{1 b i t}=2.0$} \\
\hline \multicolumn{2}{|c|}{ minimal allowed pump power } & \multicolumn{2}{|c|}{$1+\mathcal{J}+\mathcal{J}_{\mathcal{M}}{ }^{\text {min }}=0.0$} \\
\hline \multicolumn{2}{|c|}{ maximal allowed pump power } & \multicolumn{2}{|c|}{$1+\mathcal{J}+\mathcal{J}_{\mathcal{M}}{ }^{\max }=6.0$} \\
\hline \multicolumn{4}{|c|}{ Optimization constraints and how they are met } \\
\hline \multirow{5}{*}{\multicolumn{2}{|c|}{$\begin{array}{l}\text { fixed } s(0)\left(\text { either } s_{\text {on }} \text { or } s_{\text {off }}\right) \\
\text { fixed } s(T)\left(\text { either } s_{\text {off }} \text { or } s_{\text {on }}\right) \\
\frac{\partial}{\partial t} \mathcal{J}_{\mathcal{M}}(0)=0 \\
\frac{\partial}{\partial t} \mathcal{J}_{\mathcal{M}}(T)=0 \\
0<1+\mathcal{J}+\mathcal{J}_{\mathcal{M}}(\tau)<6\end{array}$}} & \multirow{5}{*}{\multicolumn{2}{|c|}{$\begin{array}{c}y(0)=p(0)=\ln (s(0)) \\
y(T)=p(1)=\ln (s(T)) \\
\dot{y}(0)=\ddot{y}(0)=\dddot{y}(0)=0 \\
\dot{y}(T)=\ddot{y}(T)=\dddot{y}(T)=0 \\
\text { active constraint }\end{array}$}} \\
\hline & & & \\
\hline & & & \\
\hline & & & \\
\hline & & & \\
\hline \multicolumn{4}{|c|}{ Results of optimization } \\
\hline & $\mathrm{N}=2$ & $\mathrm{~N}=20$ & Theory \\
\hline up transition & $T=1.80$ & $T=1.57$ & $T_{o p t}=1.42$ \\
\hline down transition & $T=1.98$ & $T=1.71$ & $T_{o p t}=1.53$ \\
\hline
\end{tabular}

relax to the steady state value.

In Fig. 2 we show the current and photon density that were obtained with just two sinusoidal terms $(\mathrm{N}=2$ in (6) $)$. The minimal transition time that can be obtained decreases when the number of base functions is increased, e.g. tenfold $(\mathrm{N}=20)$ as shown in Fig. 2 $\mathrm{b}$. The inclusion of higher frequency sine functions results in a current with a much faster rise time. Just by the choice of the number of base functions one can therefore approximately take into account limitations on the possible rise-times of electronics involved. Thus, we did not explicitly add this limitation as an additional constraint in the optimization procedure, but such a constraint is reasonable to use.

The waveforms found also converge to the theoretical best limit (Fig. 2), obtained by applying results from optimal control theory. If one drops the requirement of finite rise times for the drive current, that is, if discontinuous functions are admitted, then the so called Minimum Principle [25] can be applied. It tells us that a time-optimal state-space trajectory is achieved by driving the laser with a constant current at either the maximal allowed or minimal possible value (see as well [20]). The point in state-space where to switch from one value to the other can be found by integration of the Hamiltonian corresponding to (4) (for details see [26]). The resulting minimum transition time is a lower bound benchmark for the transition times that can be achieved with realistic input currents. It also is the lower bound for the bit period $T_{b i t}=T$ for which communication without laser induced inter-symbol interference is possible. Figure [2] shows that the results we obtain with our technique converge both in the shape of the waveform and in the value of the transition time to the optimalcontrol theory result as more base functions are included in the optimization routine.

The down transition driving the laser from the "on" power level to the "off" power level (Fig. 2 $\mathrm{k}$, e, and f) we design exactly as before except that values used for the 
boundary conditions $s(0)$ and $s(T)$ are reversed from the low to high transition. The minimal transition times are displayed in table $\amalg$ where we show the results using two and twenty base functions as well as using optimal control theory. It is important to note that the down transition is not just a time reversed version of the up transition, and that both the transition times and the waveforms are different and have to be designed separately. Most communication schemes are evenly clocked, the time necessary to transmit a symbol is independent of the particular information. Yet our results show that the fundamental dynamics of the laser may mean that approaching the optimal limits might imply using unequal symbol times. We offer this as an idea to explore but we do not pursue it further.

\section{B. Performance}

Using shaped input currents will result in a better BER performance for high bit rates because we avoid errors caused by inter-symbol interference due to the internal dynamics of the laser. To show the possible improved communication performance we compare NRZ bits obtained using the up and down transitions that were designed by the presented technique using two base functions ( $\mathrm{N}=2$ in (6) to unshaped current modulation.

A random sequence of bits is to be transmitted in this numerical experiment. The bit sequence determines the shaped current, shown in Fig 3 , which enters as driving term the laser rate equations (4). The output $s(\tau)$ is obtained by integration of (4) and Fig 3b shows that $s(\tau)$ switches, as was the design goal, between the "on" and "off" state without any relaxation oscillations. Transmitting 50 bits results in the state-space trajectory presented in Fig 3 , (thick line). The two fixed points (square and diamond) and the optimal control trajectory (dashdotted line) obtained by using non-continuous currents are also shown.

The bit time for all the panels in Fig 3 was set to $T_{b i t}=2.0$. We extend each transition to this time by appending the appropriate interval of fixed-point current. Since we normalized time using $\omega_{R}=2 \pi \mathrm{f}_{r}$ this implies that we communicate using NRZ bits that last about one third of the relaxation oscillation period $1 / \mathrm{f}_{r}$ of the fixed point $\left(S_{0}, N_{0}\right)$.

We compare the bit errors using shaped currents to squarelike current modulation where, basically, the current follows the NRZ bits but with finite rise times, to be slightly more realistic. The up and down transitions of the square-like driving current shown in Fig 31 was achieved using 'tanh' functions. The maximum slope of the current equals the maximum slope of the shaped current shown in Fig 3 h. Since the bit time is short compared to the period of the laser's intrinsic relaxation oscillations, the state-space trajectory due to the modulation of 50 NRZ-bits is complicated and exhibits large excursions (see Fig 3). Since the system does not reach either one of the two fixed points at the end of a bit-period, there is considerable dynamic coupling from past to future and hence inter-symbol interference. In Fig 3 , we show the output corresponding to the drive current above (Fig $3 \mathrm{~d}$ ). It is apparent that even without channel noise the receiver will not a)
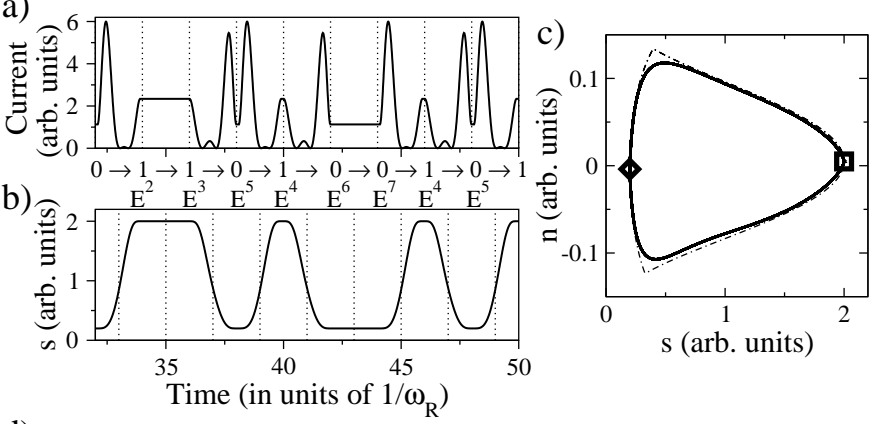

d)
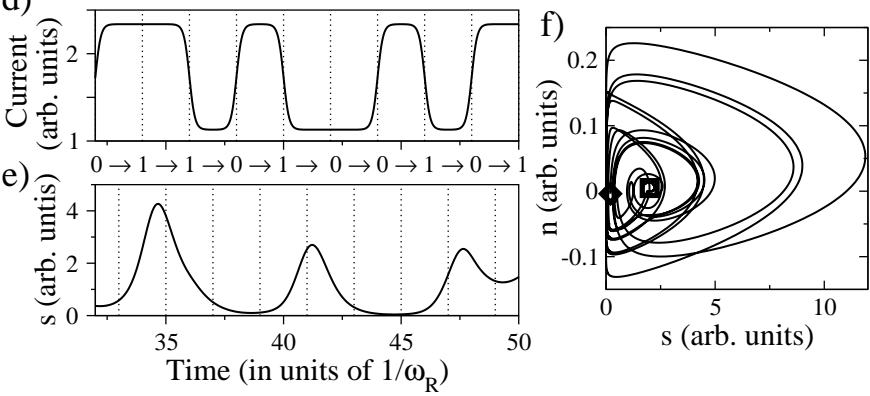

Fig. 3. Comparison between shaped current modulation (a-c) and squarelike currents (d-f). Shaped drive currents (a) result in an optical laser output (b) free of relaxation oscillations. For visual guidance, bit times are indicated by dotted lines. Note that the currents were designed to optimize the transition between the two logical states ("on" and "off") and consequently, in the output, the assigned bits appear shifted by half a bit time. The bit assignment is shown between panels a) and b) together with the corresponding bit energies (see text). Square-like currents (d) result in an output (e) corrupted by strong inter-symbol interference. Panels c) and f) are the corresponding state-space plots (see text for further details).

be able to infer without error the bit sequence that was to be transmitted.

To quantify the possible improved communication performance we calculated bit error rates (BER). For illustration, a simple additive white Gaussian noise corrupted a dispersionless channel. The overall conclusions drawn will apply to more realistic channel models, since the performance enhancement is due to avoiding inter-symbol interference in the laser and should be largely independent of the characteristics of the communication channel.

Figure 4 shows in the main panel the SNR versus the BER for high bit rate $\left(T_{b i t}=2\right)$ square-like current modulation as squares and high bit rate shaped current modulation as circles. Low bit rate $\left(T_{b i t}=8\right)$ square-like current modulation (squares) and low bit rate shaped current modulation (circles) is presented in the inset of Fig. 4 These are results of numerical experiments where one million random bits generated a corresponding input current (either shaped or square-like) to (4), which was integrated. To the resulting laser output an appropriate amount of channel noise was added. The bits were decoded at the receiver by integrating the received power over each bit time (assuming a perfect clock) and by distinguishing zero and one bits through simple thresholding. This way an estimate of the BER was obtained for each noise level.

For the case of square-like current modulation the threshold used for bit detection was determined numerically. It was optimized for each noise level separately. Furthermore, due to 


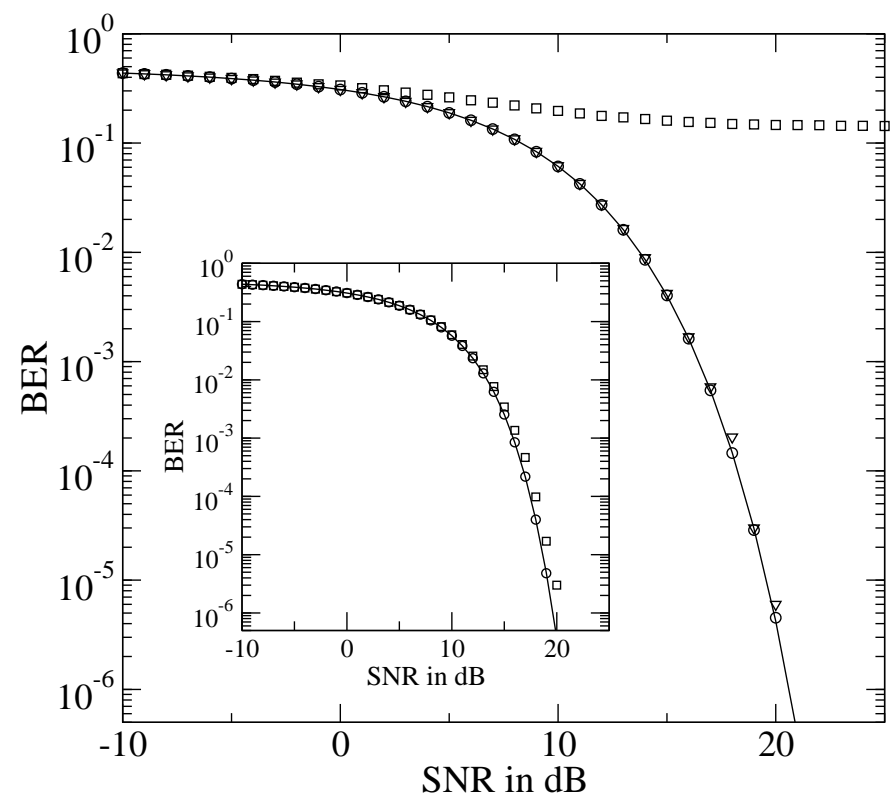

Fig. 4. The signal-to-noise ratio (SNR) versus the bit-error-rate (BER) is presented for the laser being driven by both shaped currents (circles and lines) and square-like currents (squares). The main panel shows high bit rate $\left(T_{b i t}=2\right)$ and the inset lower bit rate $\left(T_{b i t}=8\right)$ communication. The symbols represent results obtained by numerical integration and the lines are theoretical curves. We display as triangles results where stimulated emission noise is simulated.

the complex dynamics of $s(\tau)$, it is not immediately obvious when to start the receiver clock that determines the beginning and end of a bit (see Fig. 3 ). Therefore the optimal starting time was numerically estimated as well.

For a shaped current input, a simple theoretical expression for the expected BER can be derived. Consider transmission without channel noise first. Without any noise the receiver detects four different bit energies $E^{i}(i \in\{1,2,3,4\})$ signifying one bits. $^{2}$ The largest energy $E^{1}$ corresponds to a one bit being both preceded and succeeded by a one bit, the smallest energy $E^{4}$ corresponds to a one bit being both preceded and succeeded by a zero bit, whereas $E^{2}$ and $E^{3}$ correspond to one bits that contain an up or down transition (see Fig. 3). Similarly, there are four energy levels $\left(E^{j}\right.$ with $j \in\{5,6,7,8\})$ representing zero bits. The expected bit error rate is obtained by evaluating the probability that the energy that results after adding white Gaussian noise $\xi$ with variance $\sigma^{2}$ to a zero bit energy level exceeds the threshold $E_{t h}$ and by considering the probability that $E^{i}+\xi<E_{t h}$ for $i \in\{1,2,3,4\}$. Doing this the theoretically expected BER can be written in terms of the complementary error function:

$$
B E R=\frac{1}{8} \sum_{i=1}^{8} \frac{1}{2} \operatorname{erfc}\left(\frac{\left|E^{i}-E_{t h}\right|}{\sqrt{2} \sigma}\right) .
$$

The resulting curves are plotted as lines in Fig 4 where a fixed threshold given by

$$
E_{t h}=\frac{1}{2}\left[\max _{j \in[5 \ldots 8]}\left(E^{j}\right)+\min _{i \in[1 \ldots 4]}\left(E^{i}\right)\right]
$$

\footnotetext{
${ }^{2}$ For the purpose of the numerical experiment we use $E=\int_{0}^{T_{b i t}} s(t) d t$ for the bit energies because the photon density $s(t)$ is proportional to the laser output power.
}

was used.

Calculating the theoretically expected BER using the optimal threshold value $E_{t h}^{o p t}(\sigma)$, which can be derived from (7), and which is a function of the noise strength, results in a curve that to the eye is indistinguishable from the one displayed in Fig 4 For this reason we used the simpler $E_{t h}$ given by (8) in the numerical experiment determining the BER when using shaped modulation currents.

The following conclusion can be drawn from the results presented in Fig. 4 As expected, using shaped current modulation there is a very good agreement between the theoretical and numerical result for both high and low bit rates, showing that all errors are due to Gaussian channel noise. In contrast, Fig. 4 shows that high bit rates $\left(T_{b i t}=2\right)$ for square-like current modulation lead essentially to a communication breakdown. Even for very small noise levels (high SNR) on average about 14 bits per 100 transmitted bits are wrongly decoded by the receiver. These errors are caused by inter-symbol interference due to the laser dynamics. For lower bit rates, that is bit rates smaller than $\omega_{r} / 2 \pi$, the bit errors attributable to the laser dynamics become more and more negligible, as is indicated by the inset of Fig. 4 which shows that for lower bit rates the performance enhancement due to the use of shaped input currents is minor.

\section{Noise and Parameter Mismatch}

Semiconductor laser diodes are inherently noisy. To study the effects of spontaneous emission noise on the proposed communication scheme we conduct numerical experiments, where rate equations that include appropriate stochastic terms (Gaussian white noise) are driven by the shaped current, which, as discussed above, was calculated based on the deterministic model (4). The rate equation including spontaneous emission terms and a description of the integrator can be found in [22], [26].

For realistic values of the noise variance (same as in [22]) the performance advantage achieved by modulating the laser with shaped input currents persists. Although noise causes the state-space trajectory to deviate from the ideal trajectory, the deviations remained for the bit sequences and noise realization of our numerical experiments small enough to cause only slight performance degradations. The BER performance calculated in one such numerical experiment is shown as triangles in the main panel of Fig. 4 It is close to the theoretical curve calculated for the noise free system and far better than squarelike modulation of a laser model free of spontaneous emission noise.

The shaped current waveform that is used to achieve high bit rate communications without inter-symbol interference was calculated based on the model (4), the parameters of which will be known only approximately for a given laser diode. It is therefore important to consider the effects of parameter mismatch between the model used to generate the shaped driving current and the model used to numerically evaluate the BER performance.

Figure 5] shows the degradation of the BER performance for differential gain mismatches, defined as $\Delta=\left(\gamma_{n}{ }^{\prime}-\gamma_{n}\right) / \gamma_{n}{ }^{\prime}$. 


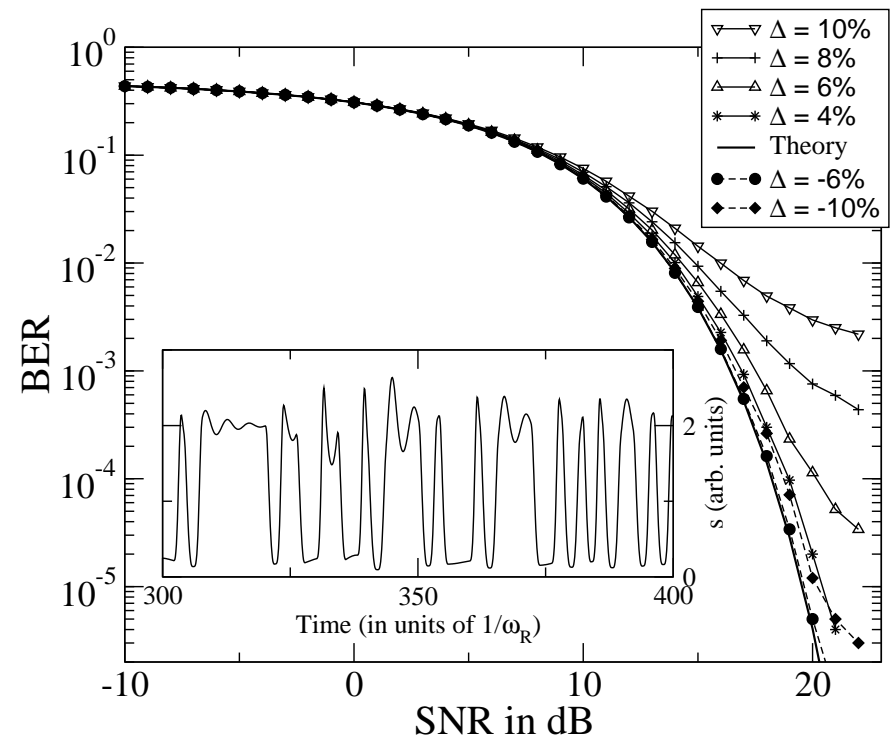

Fig. 5. BER performances for mismatches $\Delta=\frac{\gamma_{n}{ }^{\prime}-\gamma_{n}}{\gamma_{n}{ }^{\prime}}$, where $\gamma_{n}{ }^{\prime}$ is the estimated and $\gamma_{n}$ the actual differential gain. The inset shows a time trace of the photon density $s$ for $\Delta=-10 \%$ at the output of the laser. The bits are clearly distinguishable.

Here $\gamma_{n}^{\prime}$ denotes the estimated differential gain, the one used in the generation of the shaped drive current, and $\gamma_{n}$ is the actual parameter, the one characterizing the system driven by the shaped currents. Overestimating of the differential gain $(\Delta>0)$ severely degrades performance for mismatches $|\Delta|>$ $5 \%$, whereas underestimation $(\Delta<0)$ has a far less noticeable effect.

The reason is that overestimating the differential gain implies an overestimation of $\omega_{R}$, i.e. the response time of the actual laser is slower than was assumed. The laser will have slower rise and fall times and consequently a lower bit energy for a one bit that is both preceded and succeeded by a zero bit. Although the mean bit energy does not change significantly, the energy difference between the maximum energy for a zero bit $\left(\max _{j \in[5 \ldots 8]}\left(E^{j}\right)\right)$ and the minimum energy for a one bit $\left(\min _{i \in[1 \ldots 4]}\left(E^{i}\right)\right)$ is decreased resulting in a higher BER.

A similar asymmetric performance degradation holds for mismatches in the photon decay parameter $\gamma_{c}$. Performance is most sensitive to mismatches of $\gamma_{c}$ and $\gamma_{n}$ and least sensitive to mismatches in $\gamma_{p}$.

The unexpected asymmetry of the performance degradation with estimation may suggests that, in practice, it may be wiser to solve for state-space trajectories using slightly smaller values of $\gamma_{c, n}$ than the best available experimental estimations of the parameters. This may reduce the risk to performance from fluctuations in $\gamma_{c, n}$.

\section{DISCUSSION}

We demonstrate a technique to control the laser's nonlinear internal degrees of freedom to move along a specific statespace trajectory by designing current input waveforms. This can be used to optimize the optical intensity waveforms emitted to fit certain desired characteristics.

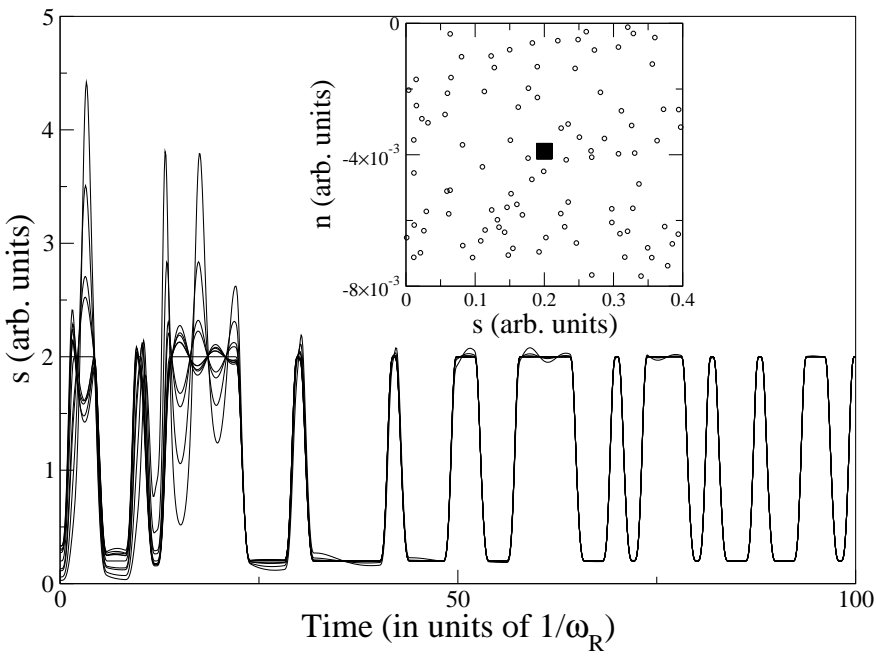

Fig. 6. Evolution of the photon density $s(t)$ in time for initial conditions chosen randomly in the neighborhood of the correct initial state (a subset of ten realizations is displayed, no noise and no mismatch). Inset: Initial conditions in state-space (circles) and where the trajectories in state-space end up (squares) after driving currents for 49 random bits were applied.

On the example of time-optimized NRZ bits, the possible BER performance enhancement due to the elimination of intersymbol interference is demonstrated. We furthermore show that spontaneous emission noise and parameter mismatches can be tolerated suggesting that this scheme can be used in practice to enhance the communication data rate when directly modulated lasers are used as transmitters.

Our numerical experiments show that including spontaneous emission noise and small but finite parameter mismatches leads to trajectories in state-space that are close to the ideal one. This suggest that the trajectory we obtain in the optimization is robust with respect to perturbations. In numerical investigations it appears that the state-space trajectory is also stable in the sense that, starting from slightly perturbed initial conditions, trajectories converge to the ideal one, when the system is driven by the same bit sequence. The result of one such numerical experiment is shown in Fig. 6 where initial relaxation oscillations soon disappear as the trajectories converge.

Theoretically, an open dynamical-systems question is whether there is some kind of easily computable generalized stability property that would quantify whether the controlled system is stable even under arbitrary message driving. It would be desirable to build this property into the optimization target to ensure that the solutions found are also stable. In our model it was fortuitous that all solutions were stable with the parameters we used, but this was not guaranteed by the method, and at present the best check is empirical integration under perturbations. We also speculate that the relative advantage of dynamically-aware current-shaping over conventional direct modulation could be significantly larger if, instead of transmitting binary symbols (two energy levels), the system was controlled to a higher alphabet. The net result of our pulse shaping is to radically reduce that part of variance which is due to dynamics in the optical intensities of the different received symbols. If the fluctuations from 
inter-symbol dynamics are eliminated, leaving only detector noise and spontaneous emission, then it is conceivable that one could transmit with more than two distinct optical levels for the symbols, and still reliably decode them. This would improve useful data rate versus binary transmission even without increasing the rate of symbols being transmitted.

The key technological issue is whether the proposed directmodulation scheme using shaped currents can be implemented at low cost. Existing technology has been developed for clock equalization, generating fast fronts at gigahertz speeds which could subsequently be shaped using delay line techniques [27]. Thus, current shaping is in principle electrically achievable. We speculate that the laser and the current shaping electronics could be integrated on one chip so that the overall cost should be comparable to that of traditional single-mode-lasers. We hope that the potential of current-shaping to mitigate problems that have plagued the direct-modulation approach to highspeed communication will stimulate further research and lead to practical applications.

\section{REFERENCES}

[1] C. H. Lee, T. H. Yoon, and S. Y. Shin, "Period doubling and chaos in a directly modulated laser diode," Appl.Phys.Lett., vol. 46, no.1, pp. 95-97, Jan. 1985

[2] Y. C. Chen, H. G. Winful, and J. M. Liu, "Subharmonic bifurcations and irregular pulsing behaviour of modulated semiconductor lasers," Appl.Phys.Lett., vol. 47, no.3, pp. 208-210, Aug. 1985

[3] M. Tang and S. Wang, "Simulation studies of bifurcation and chaos in semiconductor lasers," Appl.Phys.Lett., vol. 48, no.14, pp. 900-902, April 1986

[4] G. P. Agrawal, "Effect of gain nonlinearities on period doubling and chaos in directly modulated semiconductor lasers", Appl.Phys.Lett., vol. 49, no.16, pp. 1013-1015, Oct. 1986

[5] W. Lauterborn and R. Steinhoff, "Bifurcation structure of a laser with pump modulation," J. Opt. Soc. Am. B, vol. 5, no. 5, pp. 1097-1104, May 1988

[6] H. Lamela, G. Carpintero, and F. J. Mancebo, "Period tripling and chaos in the dynamic behavior of directly modulated diode lasers," IEEE J. Quantum Electron., vol. QE-34, no.10, pp. 1797-1801, Oct. 1998

[7] K. A. Corbett and M. W. Hamilton, "Comparison of the bifurcation scenarios predicted by the single-mode and multimode semiconductor rate equations," Phys. Rev. E, vol. 62, no.5, pp. 6487-6495, Nov. 2000

[8] H. F. Liu and W. F. Ngai, "Nonlinear dynamics of a directly modulated $1.55 \mu \mathrm{m}$ InGaAsP distributed feedback semiconductor laser," IEEE J. Quantum Electron., vol. QE-29, no.6, pp. 1668-1675, June 1993

[9] P. Colet, C. R. Mirasso, and M. S. Miguel, "Memory Diagram of SingleMode Semiconductor Lasers", IEEE J. Quantum Electron., vol. QE-29, pp. 1624-1630, June 1993

[10] K. Petermann, Laser Diode Modulation and Noise (Kluwer, Tokyo, 1988)

[11] K. Y. Lau and A. Yariv, "Ultra-High Speed Semiconductor Lasers", IEEE J. Quantum Electron., vol. QE-21, pp. 121-138, Feb. 1985

[12] R. S. Tucker, "High-Speed Modulation of Semiconductor Lasers", $J$. Lightwave Technol., vol. LT-3, pp. 1180-1192, Dec. 1985

[13] S. Riyopoulos, "Elimination of transient vertical-cavity surface-emitting laser oscillations using photoactive feedback", Appl. Phys. Lett., vol. 75 , pp. 3057-3059, Nov. 1999

[14] C. R. Mirasso, E. Hernandez-Garcia, J. Dellunde, M. C. Torrent, and J. M. Sancho, "Analytical calculations of switch-on time and timing jitter in diode lasers subjected to optical feedback and external light injection", Opt. Comm., vol. 115, pp. 523-527, April 1995

[15] Y. Suematsu and T. H. Hong "Suppression of Relaxation Oscillation in Light Output of Injection by Electrical Resonance Circuit", IEEE J. Quantum Electron., vol. QE-13, pp. 756-762, Sept. 1977

[16] M. Danielsen, "A Theoretical Analysis for Gigabit/Second Pulse Code Modulation of Semiconductor Lasers", IEEE J. Quantum Electron., vol. QE-12, pp. 657-660, Nov. 1976
[17] P. Torphammar, R. Tell, H. Eklund, and A. R. Johnston, “ Minimizing Pattern Effects in Semiconductor Lasers at High Rate Pulse Modulation", IEEE J. Quantum Electron., vol. QE-15, pp. 1271-1276, Nov. 1979

[18] R. Olshansky and D. Fye, "Reduction of dynamics linewidth in singlefrequency semiconductor lasers", Electron. Lett., vol. 20, pp. 928-929, Oct. 1984

[19] L. Bickers and L. D. Westbrook, "Reduction of transient laser chirp in $1.5 \mu \mathrm{m}$ DFB lasers by shaping the modulation pulse", IEE Proceedings, Pt. J, vol. 133, pp. 155-162, April 1986

[20] N. Dokhane and G. L. Lippi, "Improved direct modulation technique for faster switching of diode laser", IEE Proc.-Optoelectron., vol. 149, pp.7-16, Feb. 2002

[21] J. M. Liu and T. B. Simpson, "Four-Wave Mixing and Optical Modulation in a Semiconductor Laser", IEEE J. Quantum Electron., vol. QE-30, pp. 957-965, April 1994

[22] H. D. I. Abarbanel, M. Kennel, L. Illing, S. Tang, H.F. Chen and J.M. Liu, "Synchronization and Communication Using Semiconductor Lasers With Optoelectronic Feedback", IEEE J. Quantum Electron., vol. QE37, pp. 1301-1311, Oct. 2001

[23] P.E. Gill, W. Murray, M.A. Saunders, and M.H. Wright, "Some theoretical properties of an augmented Lagrangian merit function", Advances in Optimization and Parallel Computing, P.M. Pardalos, ed., North Holland, 1992, pp. 101-128.

[24] P.E. Gill, W. Murray, M.A. Saunders, and M.H. Wright, "User's Guide for NPSOL (Version 4.0): A Fortran package for nonlinear programming", Report SOL 86-2, Department of Operations Research, Stanford University, Stanford, CA, 1986

[25] M. Athans and P. L. Flab, Optimal Control: An Introduction to the Theory and Its Applications, McGraw-Hill Book Company, 1966

[26] L. Illing, Chaos synchronization and communication in semiconductor lasers, Thesis (Ph.D.), University of California, San Diego, 2002

[27] W. J. Dally, J. Poulton, "Transmitter equalization for 4-Gbps signaling", IEEE Micro, vol. 17, pp. 48-56, Jan. 1997

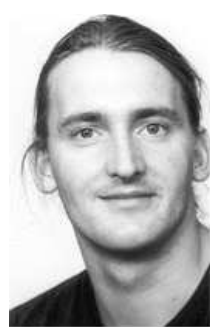

Lucas Illing was born in Halle, Germany, in 1973. He received his Vordiplom in 1996 from the Humboldt University in Berlin, Germany and in 2002 the Ph.D. in Physics from the University of California, San Diego. He is a Research Assistant at Duke University. His research is primarily concerned with chaos synchronization, control, and communication using chaos.

Matthew B. Kennel was born in Santa Monica, California in 1968. He received the A.B. degree in physics in 1989 from Princeton University and in 1995 the Ph.D. in physics from the University of California, San Diego. After a Department of Energy postdoctoral fellowship at the Oak Ridge National Laboratory, he joined the Institute for Nonlinear Science at UCSD as an Assistant Research Scientist. His research interests are in chaotic communication and the application of information theoretical algorithms to observed nonlinear dynamics. 